\title{
Impact of obesity and metabolic health status in the development of non-alcoholic fatty liver disease (NAFLD): A United Kingdom population-based cohort study using the health improvement network (THIN)
}

A. Vusirikala ${ }^{1}$, T. Thomas $^{1,2,3,4} \mathbb{B}$, N. Bhala ${ }^{1,5}$, A. A. Tahrani ${ }^{1,6,7 \dagger}$, G. N. Thomas ${ }^{1 *+}$ and K. Nirantharakumar ${ }^{1,8^{*+}}$

\begin{abstract}
Background: With the obesity epidemic reaching crisis levels, there has been attention around those who may be resilient to the effects of obesity, termed metabolically healthy obesity $(\mathrm{MHO})$, who initially present without associated metabolic abnormalities. Few longitudinal studies have explored the relationship between $\mathrm{MHO}$ and non-alcoholic fatty liver disease (NAFLD), which we address using over 4 million primary care patient records.

Methods: A retrospective population-based longitudinal cohort was conducted using The Health Improvement Network (THIN) database incorporating adults with no history of NAFLD or alcohol excess at baseline. Individuals were classified according to BMI category and metabolic abnormalities (diabetes, hypertension and dyslipidaemia). Diagnosis of NAFLD during follow-up was the primary outcome measure. NAFLD was identified by Read codes.

Results: During a median follow-up period of 4.7 years, 12,867 (0.3\%) incident cases of NAFLD were recorded in the cohort of 4,121,049 individuals. Compared to individuals with normal weight and no metabolic abnormalities, equivalent individuals who were overweight, or obese were at significantly greater risk of incident NAFLD (Adjusted HR 3.32 (95\% Cl 2.98-3.49), and 6.92 (6.40-7.48, respectively). Metabolic risk factors further increased risk, including in those with normal weight and $1(2.27,1.97-2.61)$ or $=<2(2.39,1.99-2.87)$ metabolic abnormalities.

Conclusions: $\mathrm{MHO}$ individuals are at greater risk of developing NAFLD compared to those with normal weight. This finding supports that the MHO phenotype is a temporary state, and weight must be considered a risk factor even before other risk factors develop. Being normal weight with metabolic abnormalities was also associated with risk of NAFLD.
\end{abstract}

\footnotetext{
* Correspondence: tom.thomas@nhs.net; K.Nirantharan@bham.ac.uk

${ }^{\dagger}$ A. A. Tahrani, G. N. Thomas and K. Nirantharakumar contributed equally to this work.

${ }^{1}$ Institute of Applied Health Research, University of Birmingham, Edgbaston, Birmingham B15 2TT, UK

Full list of author information is available at the end of the article
}

(c) The Author(s). 2020 Open Access This article is licensed under a Creative Commons Attribution 4.0 International License, which permits use, sharing, adaptation, distribution and reproduction in any medium or format, as long as you give appropriate credit to the original author(s) and the source, provide a link to the Creative Commons licence, and indicate if changes were made. The images or other third party material in this article are included in the article's Creative Commons licence, unless indicated otherwise in a credit line to the material. If material is not included in the article's Creative Commons licence and your intended use is not permitted by statutory regulation or exceeds the permitted use, you will need to obtain permission directly from the copyright holder. To view a copy of this licence, visit http://creativecommons.org/licenses/by/4.0/ The Creative Commons Public Domain Dedication waiver (http://creativecommons.org/publicdomain/zero/1.0/) applies to the data made available in this article, unless otherwise stated in a credit line to the data. 


\section{Background}

Non-alcoholic fatty liver disease (NAFLD) represents a major global healthcare challenge in the twenty-first century. Driven by the obesity epidemic [1], the global prevalence of NAFLD is estimated at around $25.2 \%$, and ranges from $13.5 \%$ in Africa to as high as $31.8 \%$ in the Middle East [2, 3]. However, it is well established that there is significant variation in the presence of concurrent metabolic abnormalities such as hyperglycaemia, hypertension and hyperlipidaemia in patients with obesity. This has led to the characterisation of patients with obesity and associated metabolic abnormalities into subphenotypes. Patients with obesity, as defined by body mass index (BMI), may present without the aforementioned metabolic complications. This subpopulation has been described as "metabolically healthy obese" (MHO). In contrast, a subgroup of normal BMI individuals with metabolic abnormalities have been termed "metabolically unhealthy non-obese" (MUNO) [4,5]. The implications for the development of NAFLD across these subphenotypes remains unclear.

Data from a recent large population-based cohort study (comprising $>3$ million individuals) examining cardiovascular risk across obesity phenotypes have suggested that individuals with $\mathrm{MHO}$ phenotype were at higher risk of coronary heart disease (CHD), cerebrovascular disease and heart failure than "metabolically healthy normal weight" individuals [6]. The study specifically highlights that individuals with obesity but without other metabolic abnormalities were at risk of cardiovascular events, and also that individuals with risk factors regardless of BMI status were equally at increased risk, highlighting that clinicians should be aware of the risk of cardiovascular disease in patients without obesity. Similar studies investigating the development of NAFLD have led to inconclusive results $[7,8]$.

The majority of research to date demonstrating an association between MHO and NAFLD is from one particular cohort called the Kangbuk Samsung Health Study in South Korea. First, a study by Chang et al. [7] showed a graded dose-response relationship with increasing baseline BMI in individuals with no metabolic abnormalities and incidence of NAFLD, suggesting that obesity has a major impact on the development of NAFLD irrespective of metabolic health. This study was only limited to metabolically healthy participants. However, another smaller study ( $n=3045)$ including participants from the same Korean cohort compared metabolically healthy and unhealthy individuals concluding that metabolic health is a greater determinant in developing NAFLD than obesity [8]. This study didn't use a strict definition for metabolic health and inappropriately termed individuals as metabolically healthy if they had less than two metabolic abnormalities. Studies have indicated that even having one metabolic abnormality can increase risk of cardiovascular conditions $[6,9,10]$ and therefore to accurately assess the relationship between metabolic health status and NAFLD, "metabolically healthy" should be defined as absence of any metabolic abnormality of interest.

With rates of decompensated cirrhosis and hepatocellular cancer (HCC) secondary to NAFLD estimated to double by 2030 [11] and NAFLD, a leading indication for liver transplantation (LT) [12, 13], it is increasingly important to further elucidate susceptible phenotypes for NAFLD development. Our study aimed to examine the association between different body size phenotypes with and without metabolic abnormalities and incident NAFLD.

\section{Methods}

The study is reported in line with Reporting of studies Conducted using Observational Routinely collected health Data (RECORD) guidelines [14].

\section{Study design and data source}

A retrospective population-based cohort study was conducted using data from The Health Improvement Network (THIN) database. THIN is a UK primary care database that contains anonymised electronic medical records (EMRs). As of May 2017, it includes longitudinal data on 16 million individuals (of which about 3 million are actively registered with a participating general practice at any one-time point) [15]. It covers approximately $6 \%$ of the actively registered population in the UK [15] and is representative of the UK general population in relation to age, sex, health conditions, major chronic illnesses, and mortality rates [16]. The validity and reliability of THIN data have been demonstrated for epidemiological research use [17], and has been used for studies of NAFLD [18, 19] and obesity [6].

\section{Study population}

Only adults aged 18 years or over with body mass index data (BMI) data available after their registration with the practice but before the start of the study period (Fig. 1) in the THIN database between January 1995 to May 2017 were eligible to take part in the study. To ensure only incident outcomes were captured, participants were only included in the study after they had been registered with their practice for at least 1 year. This one-year period was chosen to limit the possibility that NAFLD diagnoses documented after the patient registration date represented pre-existing disease transferred from previous medical records rather than incident cases [20].

In addition, participants were also required to be from practices that had used the Vision computer system for at least 1 year and met the criteria for acceptable 


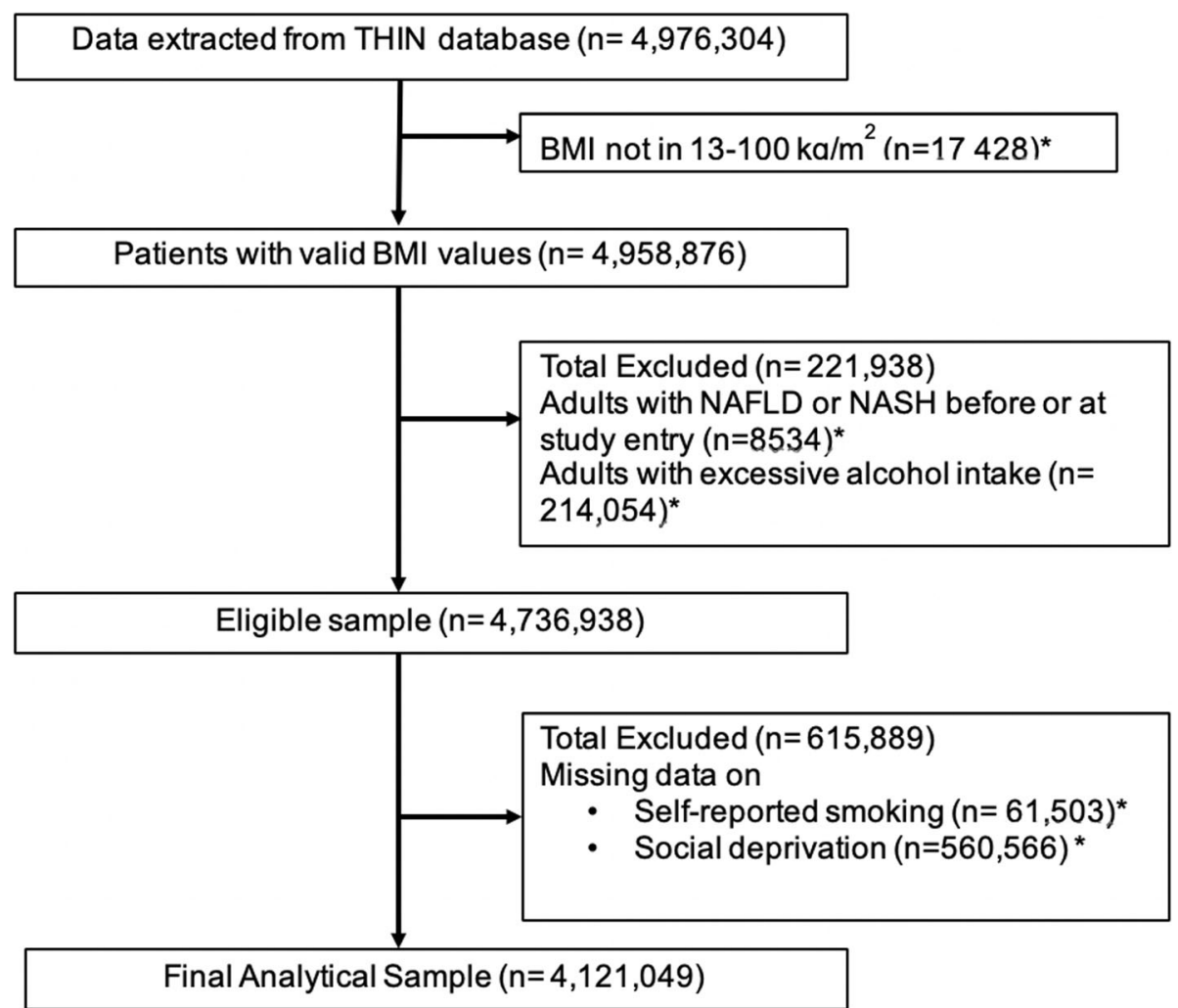

Fig. 1 Flow diagram of study population

mortality reporting (AMR) (an indicator of quality for electronic data recording) for at least 1 year preceding their study entry date.

Adults with NAFLD before or at study entry were excluded. Patients with any documentation indicative of excessive alcohol intake at baseline were excluded to ensure cases of NAFLD that may have been inaccurately diagnosed were excluded. Selection of Read codes for excessive alcohol intake was based on a previous study which used Read codes related to hazardous drinking or conditions related to hazardous drinking [19] (Appendix 1).

Of this eligible sample, patients with implausible BMI values (below $13 \mathrm{~kg} / \mathrm{m}^{2}$ or above $100 \mathrm{~kg} / \mathrm{m}^{2}$ ) were excluded [15] and to permit adjustment for deprivation and smoking, patients with missing data were excluded.

\section{Study period}

The study start date was 01 January 1995 and the study end date was 31 May 2017. The patient study entry date was the latest of the following: 12 months after patient registration date, 12 months after AMR or 12 months after Vision IT system implementation date. The study population was followed up until the first of the following events (i.e. exit date): the patient died; patient left practice, last data collection from practice or patient had a recorded diagnosis of NAFLD.

\section{Exposures}

There is no standardised definition for metabolic health or accepted criteria for distinguishing between $\mathrm{MHO}$ and (metabolically unhealthy obese) MUO for research protocols or in clinical practice. In general, most studies use BMI $>=30 \mathrm{~kg} / \mathrm{m}^{2}$ to define obesity and the absence of metabolic diseases such as diabetes, dyslipidaemia and hypertension to define "metabolically healthy" [21-23]. Therefore, in this study, individuals were grouped according to their BMI and whether they had any of those conditions [6].

\section{Metabolic abnormalities}

Diabetes and hypertension diagnoses were identified by Read code diagnoses at study entry date (Appendix 1). Recording of diabetes and hypertension in the primary care setting in the UK have been shown to be reliable [16] as they are conditions that are reported as part of the Quality Outcomes Framework which is a payment by performance scheme incentivising practices to keep an accurate register for these conditions [24]. Dyslipidaemia diagnosis was defined as those who were recorded to have been prescribed lipid-lowering medications using 
prescription codes or by laboratory measurements of elevated serum cholesterol or triglycerides or low-density lipoprotein-cholesterol (LDL-C) or low high-density lipoprotein-cholesterol (HDL-C) at baseline. The National Cholesterol Education Program (NCEP) Adult Treatment Panel (ATP) III classification of total and LDL-cholesterol were used to define high levels of total cholesterol as $>6.2 \mathrm{mmol} / \mathrm{L}(>240 \mathrm{mg} / \mathrm{dL})$ and high levels of LDL-C as $>=4.15 \mathrm{mmol} / \mathrm{L} \quad(>=160 \mathrm{mg} / \mathrm{dL})$. The ATP III triglycerides and HDL-C cut-offs for metabolic syndrome was used to define elevated triglycerides as $>=1.7 \mathrm{mmol} / \mathrm{L}(>=150 \mathrm{mg} / \mathrm{dL})$ and low HDL$\mathrm{C}$ as $<1.03 \mathrm{mmol} / \mathrm{L}(<40 \mathrm{mg} / \mathrm{dL})$ in men and $<1.29$ $\mathrm{mmol} / \mathrm{L}(<50 \mathrm{mg} / \mathrm{dL})$ in women [25].

Participants who had no recorded diabetes, hypertension or dyslipidaemia at study entry date (baseline status) but developed these metabolic abnormalities during the observation period were analysed according to their status at study entry.

\section{BMI}

BMI was categorised based on the World Health Organisation criteria: underweight (BMI of $<18.5 \mathrm{~kg} / \mathrm{m}^{2}$ ), normal weight (BMI of $18.5 \mathrm{~kg} / \mathrm{m}^{2}$ to $<25 \mathrm{~kg} / \mathrm{m}^{2}$ ), overweight (BMI of $25 \mathrm{~kg} / \mathrm{m}^{2}$ to $<30 \mathrm{~kg} / \mathrm{m}^{2}$ ), and obese (BMI of $\geq 30 \mathrm{~kg} / \mathrm{m}^{2}$ ) [26].

Baseline BMI was extracted from the dataset as the BMI recorded at time of patient registration or the first measurement when the practice was eligible to contribute towards the study (Fig. 1). This approach minimised the chance that the BMI was recorded due to particular clinical reasons but more likely to have been recorded for administrative purposes.

\section{Subgroups characterised by metabolic health status and BMI}

A metabolic abnormality score for each individual ranging from 0 to 3 was created by assigning one point each for the presence of a metabolic abnormality (diabetes, hypertension and dyslipidaemia), and categorised into 0 , 1,2 or more risk factors. These were then further stratified into 11 subgroups or phenotypes based on BMI.

\section{Outcome (dependent variable)}

The primary outcome endpoint for analysis was a recorded diagnosis of NAFLD or NASH. Diagnoses of NAFLD and NASH were defined by Read codes (Appendix 1) [19].

\section{Covariates}

Covariates considered for the analyses were age (continuous variable), sex (male or female), presence of hypothyroidism, self-reported smoking status (categorised as current; ex-smoker, never smoker) and social deprivation (categorical variable) on the patient's record at study entry.

\section{Statistical analysis \\ Main analyses}

The primary complete-case analyses included only patients with no missing or implausible data.

Incidence rates were calculated as the number of incident cases divided by person-years of follow-up. Incidence rate for NAFLD were reported by each phenotype based on BMI and metabolic status.

Cox proportional hazard models were used to assess the associations between each one of the eleven subgroups and NAFLD to derive hazard ratios (HRs) and 95\% confidence intervals (CIs). Normal weight and no metabolic abnormalities were selected as the reference category for all analyses. A hierarchical analytical approach was taken; firstly, unadjusted analyses were performed, which was subsequently adjusted for age at when BMI was recorded, sex, self-reported smoking status, and social deprivation. The proportional hazards assumption was tested by generating and visually inspecting log-log survival plots.

\section{Sensitivity analyses}

Since exclusions due to missing data may result in a selected sample, a sensitivity analysis was conducted where the available recording of Townsend score and smoking along with a missing category in the model were included. This analysis allowed for comparison of results with the results of the complete-case approach (primary analysis).

In addition, further analysis was conducted to check whether altering the definition of dyslipidaemia would alter the study conclusions. As previously noted, diabetes and hypertension are likely to be well recorded due to the QOF incentives scheme [24]. However, this is not the case for dyslipidaemia. A previous study classified dyslipidaemia by the prescription of lipid-modifying drugs only [6], therefore a sensitivity analysis was performed to assess the possibility of misclassification error due to our dyslipidaemia definition (Dyslipidaemia defined by drug lipid codes and laboratory measurements of cholesterol, triglycerides, LDL-C HDL-C vs dyslipidaemia defined by drug lipid codes only). These further analyses tested the robustness of the findings of the primary analyses.

Statistical significance was defined as $p<0.05$. All data cleaning and statistical analyses was conducted in STATA version 15.0 (Stata Corp, College Station, TX).

\section{Ethics}

The THIN organisation was granted permission to acquire and provide pseudonymised patient information to 
academics in 2003 by the National Health Service South-East Multi-centre Research Ethics Committee [27]. Authorization for this project were obtained from the University of Birmingham Scientific Review Committee (18THIN094).

\section{Results}

4,976,304 adult patients with BMI values who were registered with practice that had Vision computer system and AMR at least for 1 year between January 1995 to May 2017 were extracted from the THIN database. Figure 1 shows the derivation of the final analytical sample of 4,121,049 patients $(1,777,177$ men and 2,343,872 women). Table 1 demonstrates the baseline characteristics of study participants by BMI category and metabolic health status.

\section{Missing data}

Of the $4,736,938$ eligible patients $>=18$ years of age in the THIN database without a history of NAFLD or excessive alcohol intake at baseline, we excluded persons with missing data for smoking $(61,503$ [1.3\%]) and social deprivation $(560,566[11.8 \%])$. There was no missing data for age or sex. After these exclusions, there remained a final sample of 4,121,049 (87\% of the eligible sample). The median follow-up period for participants was 4.7 years (IQR $1.9-8.7$ years).
Stability of metabolically healthy overweight and obese Among initially overweight individuals with no metabolic abnormalities, approximately $1.2 \%$ developed diabetes, $6.8 \%$ developed hypertension and $17.3 \%$ dyslipidaemia (based on lipid prescription and dyslipidaemia READ codes) by the study end date. Among individuals who were initially $\mathrm{MHO}$, approximately $3.6 \%$ developed diabetes, $9.8 \%$ developed hypertension and $21.7 \%$ dyslipidaemia by the study end date.

\section{Incidence rate of NAFLD}

$12,867(0.3 \%)$ incident cases of NAFLD were identified during 23,254,181.4 person-years of follow up (incidence rate 0.55 per 1000 person-years). The incidence rate for NAFLD increased with each increasing BMI category and increasing number of metabolic abnormalities (Table 2) with the highest incidence rate (1.62 per 1000 person-years) seen in those who are obese with 2 or greater metabolic abnormalities.

\section{Association between metabolic health status and body size phenotype and NAFLD}

Figure 2 presents the results of unadjusted and adjusted cox regression analyses undertaken to assess the risk of NAFLD among different metabolically defined body size phenotypes. Individuals who were overweight with no metabolic abnormalities were at nearly 3.2-fold higher

Table 1 Baseline characteristics of study participants by body mass index category and metabolic health status $(n=4,121,049)$

\begin{tabular}{|c|c|c|c|c|c|c|}
\hline Characteristics & Overall & $\begin{array}{l}\text { Underweight } \\
(\boldsymbol{n}=110,084)\end{array}$ & $\begin{array}{l}\text { Normal Weight } \\
(n=1,706,932)\end{array}$ & $\begin{array}{l}\text { Overweight } \\
(n=1,377,896)\end{array}$ & $\begin{array}{l}\text { Metabolically healthy } \\
\text { and Obese } \\
(\boldsymbol{n}=466,571)\end{array}$ & $\begin{array}{l}\text { Metabolically Unhealthy }{ }^{\mathrm{a}} \\
\text { and Obese } \\
(\boldsymbol{n}=459,566)\end{array}$ \\
\hline Age median (IQR) & $44.2(30.4-60.6)$ & $29.5(26.7-56.1)$ & $37.4(26.7-56.1)$ & $48.7(34.8-63.4)$ & $39.15(29.7-50.1)$ & $58.4(48.3-67.9)$ \\
\hline \multicolumn{7}{|l|}{ Sex n (\%) } \\
\hline Males & $1,777,177(43.1)$ & $27,134(24.5)$ & $627,873(36.8)$ & $720,650(52.3)$ & $179,916(38.6)$ & $221,604(48.2)$ \\
\hline Females & $2,343,872(56.9)$ & $82,950(75.5)$ & $1,079,059(63.2)$ & $657,246(47.7)$ & $286,655(61.4)$ & $237,962(51.8)$ \\
\hline \multicolumn{7}{|l|}{ Townsend index n (\%) } \\
\hline 1 & $957,722(23.2)$ & $19,355(17.6)$ & $399,720(23.4)$ & $345,073(25.0)$ & $94,968(20.4)$ & $98,606(21.5)$ \\
\hline 2 & $868,163(21.1)$ & $19,187(17.4)$ & $351,685(20.6)$ & $306,703(22.3)$ & $93,059(19.9)$ & $97,529(21.2)$ \\
\hline 3 & $894,788(21.7)$ & $23,498(21.3)$ & $367,703(21.6)$ & $297,443(21.6)$ & $104,192(22.3)$ & $101,952(22.2)$ \\
\hline 4 & $824,753(20.0)$ & $27,044(24.6)$ & $347,132(20.3)$ & $255,910(18.6)$ & $100,596(21.6)$ & $94,071(20.5)$ \\
\hline 5 & $575,623(14.0)$ & $21,000(19.1)$ & $240,692(14.1)$ & $172,767(12.5)$ & $73,756(15.8)$ & $67,408(14.7)$ \\
\hline Missing data & 560,566 & 16,834 & 245,888 & 178,864 & 62,445 & 56,535 \\
\hline \multicolumn{7}{|l|}{ Smoking status n (\%) } \\
\hline Never smoker & $2,310,052(56.0)$ & $62,861(57.1)$ & $989,362(58.0)$ & $755,361(54.8)$ & $262,833(56.3)$ & $239,635(52.1)$ \\
\hline Ex-smoker & $875,978(21.3)$ & $12,920(11.7)$ & $290,992(17.0)$ & $333,223(24.2)$ & $94,049(20.2)$ & $144,794(31.5)$ \\
\hline Current smoker & $935,019(22.7)$ & $34,303(31.2)$ & $426,578(25.0)$ & $289,312(21.0)$ & $109,689(23.5)$ & $75,137(16.3)$ \\
\hline Missing data & 61,503 & 2640 & 24,862 & 18,869 & 10,042 & 5090 \\
\hline Hypothyroidism n (\%) & $165,477(4.0)$ & $3290(3.0)$ & $50,141(2.9)$ & $56,102(4.1)$ & $17,206(3.7)$ & $38,738(8.4)$ \\
\hline BMI $\left(\mathbf{k g} / \mathbf{m}^{2}\right)$ median (IQR) & $25.7(22.7-29.4)$ & $17.6(16.9-18.1)$ & $22.4(20.9-23.7)$ & $27.1(26-28.4)$ & $33(31.2-36.1)$ & $33.4(31.4-36.6)$ \\
\hline
\end{tabular}


Table 2 Incidence rates of NAFLD by each body size phenotype with and without metabolic abnormalities

\begin{tabular}{|c|c|c|c|c|}
\hline Body size phenotype & Sample size & Incident Cases & Person-years & $\begin{array}{l}\text { Incidence rate } \\
\text { (per } 1000 \text { person-years }\end{array}$ \\
\hline Underweight, 0 metabolic abnormalities & 94,189 & 23 & $418,897.5$ & 0.05 \\
\hline Underweight, 1 metabolic abnormality & 15,895 & 7 & $65,583.1$ & 0.11 \\
\hline Normal weight, 0 metabolic abnormalities & $1,367,321$ & 882 & $7,137,510$ & 0.12 \\
\hline Normal weight, 1 metabolic abnormality & 223,270 & 377 & $1,287,137$ & 0.29 \\
\hline Normal weight, $\geq 2$ metabolic abnormalities & 116,341 & 219 & $658,987.8$ & 0.33 \\
\hline Overweight, 0 metabolic abnormalities & 852,223 & 2112 & $4,926,580$ & 0.43 \\
\hline Overweight, 1 metabolic abnormality & 307,553 & 1437 & $1,885,388$ & 0.76 \\
\hline Overweight, $\geq 2$ metabolic abnormalities & 218,120 & 1158 & $1,350,602$ & 0.86 \\
\hline Obese, 0 metabolic abnormalities & 466,571 & 2506 & $2,727,789$ & 0.92 \\
\hline Obese, 1 metabolic abnormality & 232,795 & 1909 & $1,412,798$ & 1.35 \\
\hline Obese, $\geq 2$ metabolic abnormalities & 226,771 & 2237 & $1,382,909$ & 1.62 \\
\hline
\end{tabular}

risk of NAFLD (adjusted HR 3.23, 95\%CI 2.98-3.49) than those who were normal weight with no metabolic abnormalities. This risk continued to rise with those individuals who were obese with no metabolic abnormalities having an adjusted HR of 6.92, 95\%CI 6.40-7.48) compared to normal weight with no metabolic abnormalities (Fig. 2b).

Risk of NAFLD increased with each increasing BMI category (underweight, normal weight, overweight, obese) and within each BMI category risk increased with increasing number of metabolic abnormalities (Fig. 2b). Compared to individuals of normal weight with no metabolic abnormalities, those who were normal weight with metabolic abnormalities were associated with increased hazard of NAFLD (normal weight with one metabolic abnormality: adjusted HR 2.27, 95\%CI 1.972.61 ), normal weight with two or more metabolic abnormalities: adjusted HR 2.39, 95\%CI 1.99-2.87) (Fig. 2b). In addition, those who were underweight without metabolic abnormalities had a decreased risk of NAFLD (adjusted HR 0.50, 95\%CI 0.33-0.75).

\section{Sensitivity analysis}

Inclusion of those with missing information on social deprivation and smoking status in the regression model, did not significantly change the results (see Appendix 2, Table 1).

When dyslipidaemia was defined only by lipidmodifying drug prescriptions only, the size of the associations between body size phenotypes and metabolic status with NAFLD were slightly smaller but remained statistically significant (see Appendix 2, Table 2). The sensitivity analysis did not alter the conclusions of the study.

\section{Discussion}

This study has three key findings. First, metabolically healthy individuals with overweight or obese were at increased risk of developing NAFLD in comparison with individuals of normal weight with no metabolic abnormalities. Secondly, individuals with normal weight who were metabolically unhealthy also had increased risk of NAFLD compared to their counterparts without metabolic abnormalities. Thirdly, the presence of metabolic abnormalities with obesity potentiates the risk of NAFLD. The NAFLD risk in metabolically unhealthy individuals with overweight and obesity was markedly greater than in their counterparts without metabolic abnormalities. While the association between obesity and metabolic health with NAFLD has been examined in previous Asian cohorts, this study provides the first evidence from a cohort study characterising this relationship in a European population.

The key findings of this study concur with results of previous studies, which are predominantly crosssectional and cohort studies conducted in Asia. While the hazard ratio estimates were higher in our study in comparison to previous cohort studies conducted in China and Korea [7, 28], this may reflect differences in study populations and size. Neither of the two studies controlled for hypothyroidism, which may have biased results. In addition, the Korean retrospective cohort study was limited to only metabolically healthy participants [7], and in a relatively young population limiting the generalisability of their results. On the other hand, our study may have overestimated the effect sizes due to differentially higher surveillance in the obese population as well as the possibility that incidence cases of NAFLD likely represent a more severe phenotype as the majority of patients with NAFLD in the UK are undiagnosed.

The mechanisms underpinning the development of NAFLD are not fully understood. The traditional "two hit theory" may apply in regard to the role of obesity and metabolic abnormalities in the pathogenesis of NAFLD [29]. The "first hit" can be obesity/excess 
A. Hazard ratios (HRs) for diagnosis of NAFLD based on metabolic health status and body size phenotype. $\mathrm{HRs}$ with $95 \% \mathrm{Cl}$ are presented compared with the reference category normal weight with no metabolic abnormalities

\section{(A) Unadjusted HRs}

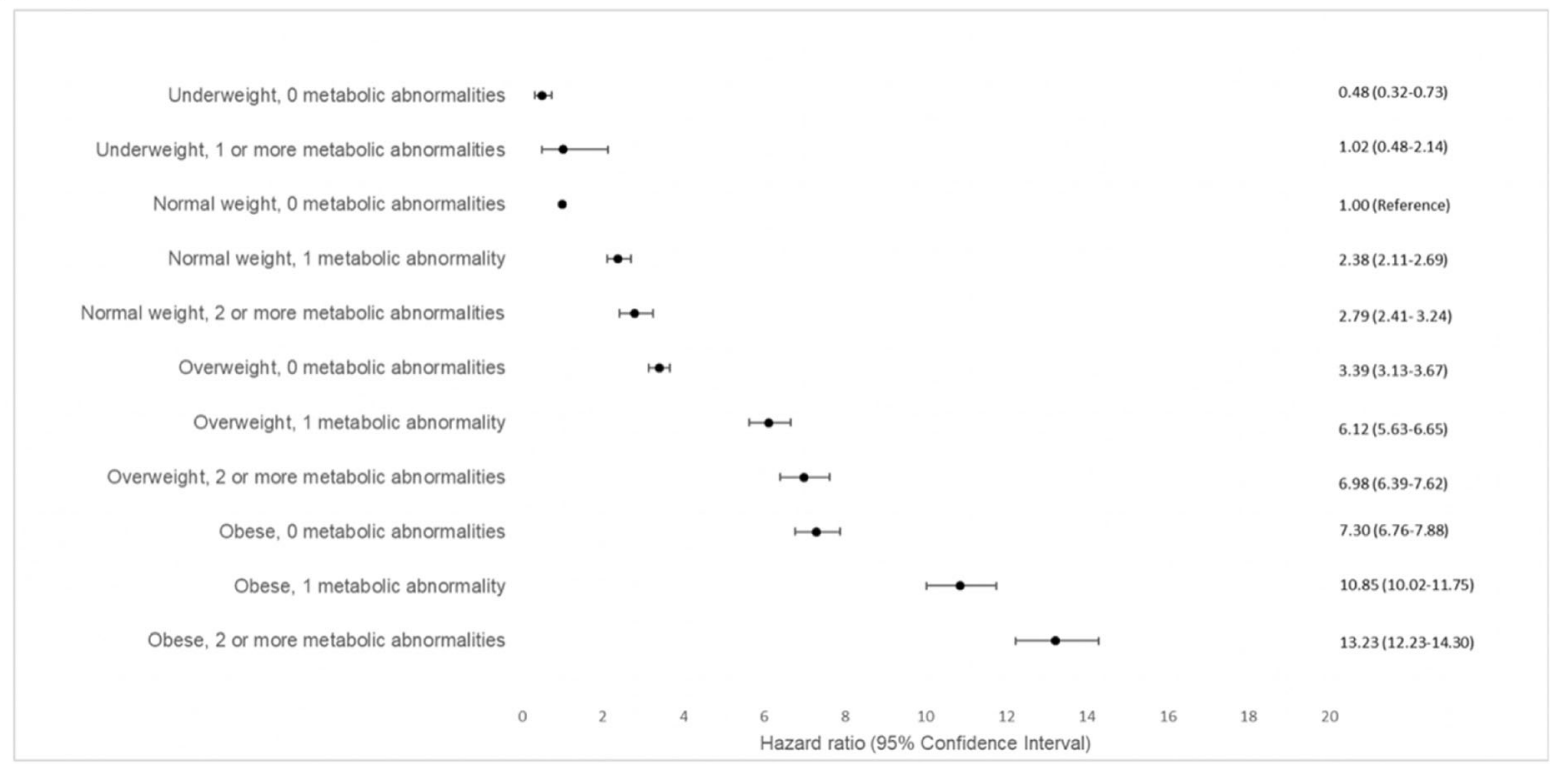

B: Multivariable-adjusted HR- adjusted for age, sex, smoking status, social deprivation, hypothyroidism at baseline.

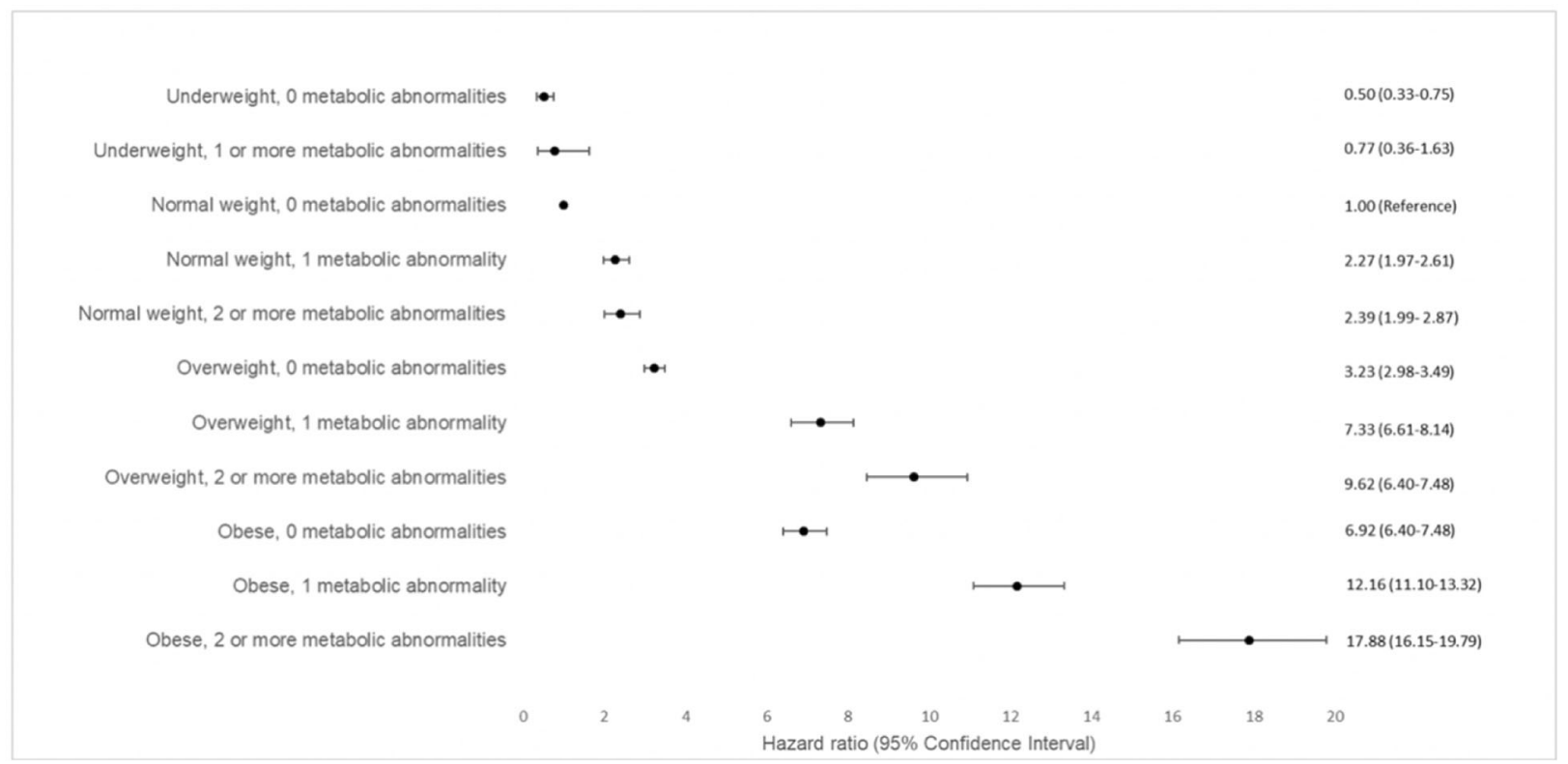

Fig. 2 a Hazard ratios for diagnosis of NAFLD based on metabolic health status and body size phenotype. HRs with 95\% Cl are presented compared with the reference category, normal weight with no metabolic abnormalities. b Multivariable-adjusted HR - adjusted for age, sex, smoking status, social deprivation, hypothyroidism at baseline

adiposity, leading to increased free fatty acid release and delivery to the liver resulting in hepatic steatosis, in turn sensitising the liver to the "second hit". Adipose tissue also releases hormones and cytokines with proinflammatory effects such as leptin, interleukin-6 and tumour necrosis factor-alpha which may lead to the development of NAFLD. Furthermore, obesity increases the risk of obstructive sleep apnoea which is associated with NAFLD [30, 31]. Metabolic abnormalities may contribute to the "second hits", such as inflammatory cytokines and oxidative stress, which result in further progression of NAFLD, to NASH and liver fibrosis/ 
cirrhosis [32]. The findings of this study demonstrate that obesity independently increases the risk of NAFLD. Although this study cannot differentiate NASH from NAFLD, it has been previously suggested that excess adipose tissue may result in NASH and liver fibrosis without the "second hit" step [33]. Moreover, a study found that the expression of genes involved in inflammation were similarly modified in metabolically healthy and unhealthy obese individuals emphasising careful interpretation is needed when considering the MHO phenotype as harmless [34]. Indeed, lifestyle modification for even non-obese individuals with NAFLD has shown to significantly improve the degree of steatosis [35].

It has been proposed that the increased risk of adverse health outcomes reported for individuals with MHO indicates that this phenotype on a population-level is a transient state prior to further progression to the MUO state [36]. This is supported by the younger age and the high incidence of the metabolic abnormalities in this group. Studies with follow-up periods ranging from 11 to 20 years further support this contention [37-39].

Being a large primary care dataset, the THIN database has enabled access to millions of patient records in the $\mathrm{UK}$, giving us unprecedented statistical power to evaluate the association between the MHO phenotype and the risk of developing incident NAFLD. To the best of our knowledge, this is the largest study to date and the first cohort study outside of Asia to look at this association. This large cohort allowed us to stratify individuals into subgroups according to the four main BMI categories and the number of metabolic abnormalities present, permitting a more detailed analysis than previously conducted studies with varying definitions of "metabolically healthy" patients. The present study used the number of concurrent metabolic abnormalities $(0,1,2$ or more) as being reflective of differing degrees of "metabolic health" in included patients. This in turn allowed us to examine whether there is a considerable impact on the risk of incident cases of NAFLD according to metabolic healthrelated co-morbidities. Additionally, a major strength of the study was being able to adjust for important baseline characteristics such as age, sex, smoking status, socioeconomic deprivation and hypothyroidism which could confound the relationship between $\mathrm{MHO}$ and the risk of NAFLD. Sample bias was also limited by excluding individuals who had a history of alcohol excess at baseline. This ensured that cases of NAFLD that may have been diagnosed in error were excluded.

Considering only the baseline BMI in included patients could potentially have led to misclassification bias. However, as previously noted by Caleyachetty et al., due to the lack of successful and established weight loss interventions, individuals are likely to remain the same weight or gain weight compared to losing weight [6].
Therefore, it can be argued that the room for misclassification when adopting this baseline BMI approach for analysis is minimal and would likely lead to an underestimation as opposed to an overestimation of the risk for NAFLD. The impact of weight changes over time on NAFLD incidence would be useful to examine in another study, however, as THIN, a routinely collected primary care database lacks has relatively limited repeated assessment of BMI in all patients, another data source may be needed. Another limitation of the present study lies in the use of BMI to define obesity. BMI does not account for central adiposity, associated increase in waist circumference and visceral fat. Indeed, one difference between MHO and MUNO could be the proportion of visceral fat in these categories. The use of magnetic resonance imaging (MRI) to characterise body fat distribution in a study on adolescents has previously linked visceral fat with impaired glucose metabolism and hepatic fat accumulation [40]. A large-scale cross-sectional study has also explored the association between visceral fat and presence of NAFLD, discerning an elevated OR of 17.813 (95\% CI 10.815-29.342) on comparing patients with normal and high level of visceral fat $\left(>100 \mathrm{~cm}^{2}\right)$ [41]. However, given that the present study is carried out on a population-based primary care database, we were unable to obtain reliable data on visceral fat or waist circumference, hence had to use BMI.

It should also be noted that the observed associations in this study may not reflect the true relationship of metabolic status and obesity with NAFLD, as NAFLD may go undetected in the UK due to lack of systematic screening. The population prevalence estimated from studies where every participant underwent an ultrasound examination was much higher $[42,43]$ than the baseline prevalence of NAFLD in this study. This underascertainment of cases may be differential between those with and without obesity. For instance, those thought to be at risk, such as those with obesity or with other metabolic abnormalities, may be preferentially screened for NAFLD. Loomis et al. noted that the true risk of NAFLD in non-obese individuals could potentially be underestimated if the diagnosis of NAFLD is not being sought in those of normal weight. This may in turn overestimate the risk of NAFLD resulting from obesity [18]. Another limitation of the presented study is that use of READ codes could potentially fail to identify all subjects with NAFLD, and it is well recognised that NAFLD remains underdiagnosed in the community. Despite this, a primary care database such as THIN that provides a recorded diagnosis of NAFLD appears to be the most appropriate method to obtain data on incident NAFLD for a large population-based epidemiological study in the UK. This study provides a useful estimate of recorded NAFLD in clinical practice in UK according to 
subgroups based on BMI and metabolic health status, reflecting "real-life" care.

However, the main results of this study are likely robust and externally valid due to the following reasons. The finding that $\mathrm{MHO}$ individuals are at higher risk of NAFLD compared to individuals of normal weight with no metabolic abnormalities is consistent with the findings of all cross-sectional and cohort studies from other settings. A large cohort study conducted in a metabolically healthy Korean population who had annual ultrasound examinations reported a 4-fold difference in NAFLD incidence rate between normal weight and obese individuals (incidence rate 20.3 per 1000 personyears in normal weight group vs incidence rate 85.9 per 1000 person-years in obese group) [7]. In addition, the unadjusted and adjusted analyses in this study examining the risk of NAFLD among individuals in the different obese subgroups resulted in large HRs above 6. The magnitude of the results in this study may suggest causality as any bias or residual confounding is unlikely to greatly attenuate the strong association observed.

\section{Conclusions}

In conclusion, individuals with $\mathrm{MHO}$ are at greater risk of developing NAFLD compared to metabolically healthy normal weight individuals. Additionally, the incidence of NAFLD increased with the increasing number of metabolic abnormalities in overweight and obese patients. These findings support that MHO phenotype is a fallacy and using the term may mislead individuals to believe obesity can be "healthy". This study has also highlighted that individuals considered to be of normal weight can have metabolic abnormalities and can be at higher risk of NAFLD. Therefore, clinicians should be aware that NAFLD can occur in this subset of normal weight individuals and may need to counsel them on management to help prevent progression to adverse liver and cardiometabolic outcomes.

\section{Supplementary information}

Supplementary information accompanies this paper at https://doi.org/10. 1186/s12902-020-00582-9.

Additional file 1: Appendix 1. Read Codes. Appendix 2. Sensitivity Analyses Table S1. Table S2. Development of NAFLD by body size phenotype and metabolic status using metabolic status derived from diagnostic codes for diabetes and hypertension and prescription records of lipid-modifying drugs only.

\section{Abbreviations}

NAFLD: Non-alcoholic fatty liver disease; MHO: Metabolically healthy obese; THIN: The Health Improvement Network; MUNO: Metabolically unhealthy non-obese; BMI: Body mass index

Acknowledgments

Nil

\section{Authors' contributions}

VA, AT, NT, KN jointly conceived the study. VA performed statistical analysis and wrote the first draft. $\Pi, N B, A T, N T, K N$ carried out subsequent revisions. The authors read and approved the final manuscript.

Funding

Nil external funding received.

\section{Availability of data and materials}

Patient data not available for sharing.

\section{Ethics approval and consent to participate}

The THIN organisation was granted permission to acquire and provide pseudonymised patient information to academics in 2003 by the National Health Service South-East Multi-centre Research Ethics Committee [27]. Authorization for this project were obtained from the University of Birmingham Scientific Review Committee (18THIN094).

Consent for publication

All authors give consent to publication.

Competing interests

Authors declare no competing interests.

\section{Author details}

${ }^{1}$ Institute of Applied Health Research, University of Birmingham, Edgbaston, Birmingham B15 2TT, UK. ${ }^{2}$ Translational Gastroenterology Unit, University of Oxford, Oxford, UK. ${ }^{3}$ Kennedy Institute of Rheumatology, University of Oxford, Oxford, UK. ${ }^{4}$ Wellcome Centre for Human Genetics, University of Oxford, Oxford, UK. ${ }^{5}$ Queen Elizabeth Hospital Birmingham, Birmingham, UK. ${ }^{6}$ Department of Diabetes and Endocrinology, University Hospitals Birmingham, Birmingham, UK. ${ }^{7}$ Centre for Endocrinology, Diabetes and Metabolism (CEDAM), Birmingham Health Partners, Birmingham, UK. ${ }^{8}$ Institute of Metabolism and Systems Research, University of Birmingham, Birmingham, UK.

Received: 3 May 2020 Accepted: 22 June 2020

Published online: 30 June 2020

References

1. Ng M, Fleming T, Robinson M, Thomson B, Graetz N, Margono C, et al. Global, regional, and national prevalence of overweight and obesity in children and adults during 1980-2013: a systematic analysis for the global burden of disease study 2013. Lancet. 2014;384(9945):766-81 [cited 2019 Mar 6]; Available from: http://www.ncbi.nlm.nih.gov/pubmed/24880830.

2. Younossi ZM, Koenig AB, Abdelatif D, Fazel Y, Henry L, Wymer M. Global epidemiology of nonalcoholic fatty liver disease-meta-analytic assessment of prevalence, incidence, and outcomes. Hepatology. 2016;64(1):73-84[cited 2018 Aug 16]. Available from:. https://doi.org/10.1002/hep.28431.

3. Karelis AD, St-Pierre DH, Conus F, Rabasa-Lhoret R, Poehlman ET. Metabolic and body composition factors in subgroups of obesity: what do we know? J Clin Endocrinol Metab. 2004;89(6):2569-75 [cited 2019 Mar 6]. Available from: http://www.ncbi.nlm.nih.gov/pubmed/15181025.

4. Ruderman NB, Schneider SH, Berchtold P. The "metabolically-obese," normal-weight individual. Am J Clin Nutr. 1981;34(8):1617-21 [cited 2019 Mar 6]. Available from: http://www.ncbi.nlm.nih.gov/pubmed/7270486.

5. Conus F, Rabasa-Lhoret R, Péronnet F. Characteristics of metabolically obese normal-weight (MONW) subjects. Appl Physiol Nutr Metab. 2007;32(6):4 [cited 2019 mar 6]. Available from: http://www.ncbi.n/m.nih.gov/pubmed/1 7332780 .

6. Caleyachetty R, Thomas GN, Toulis KA, Mohammed N, Gokhale KM, Balachandran $\mathrm{K}$, et al. Metabolically healthy obese and incident cardiovascular disease events among 3.5 million men and women. J Am Coll Cardiol. 2017;70(12):1429-37 [cited 2019 mar 6]. Available from: http:// www.ncbi.nlm.nih.gov/pubmed/28911506.

7. Chang Y, Jung H-S, Cho J, Zhang Y, Yun KE, Lazo M, et al. Metabolically healthy obesity and the development of nonalcoholic fatty liver disease. Am J Gastroenterol. 2016;111(8):1133-40 [cited 2019 mar 6]. Available from: http://www.ncbi.n/m.nih.gov/pubmed/27185080.

8. Lee MK, Rhee EJ, Kim MC, Moon BS, Lee Jl, Song YS, et al. Metabolic health is more important than obesity in the development of nonalcoholic fatty 
liver disease: a 4-year retrospective study. Endocrinol Metab (Seoul, Korea). 2015;30(4):522-30 [cited 2019 mar 6]. Available from: http://www.ncbi.nlm nih.gov/pubmed/26394730.

9. Thomsen M, Nordestgaard BG. Myocardial infarction and ischemic heart disease in overweight and obesity with and without metabolic syndrome. JAMA Intern Med. 2014;174(1):15 [cited 2019 mar 6]. Available from: http:// www.ncbi.nlm.nih.gov/pubmed/24217719.

10. Meigs JB, Wilson PWF, Fox CS, Vasan RS, Nathan DM, Sullivan LM, et al. Body mass index, metabolic syndrome, and risk of type 2 diabetes or cardiovascular disease. J Clin Endocrinol Metab. 2006;91(8):2906-12 [cited 2019 mar 6]. Available from: http://www.ncbi.nlm.nih.gov/ pubmed/16735483.

11. Estes C, Razavi H, Loomba R, Younossi Z, Sanyal AJ. Modeling the epidemic of nonalcoholic fatty liver disease demonstrates an exponential increase in burden of disease. Hepatology. 2018;67(1):123-33[cited 2019 Mar 7]. Available from:. https://doi.org/10.1002/hep.29466.

12. Charlton MR, Burns JM, Pedersen RA, Watt KD, Heimbach JK, Dierkhising RA. Frequency and outcomes of liver transplantation for nonalcoholic steatohepatitis in the United States. Gastroenterology. 2011;141(4):1249-53 [cited 2019 Mar 6]. Available from: https://www.sciencedirect.com/science/ article/pii/S0016508511009115.

13. Goldberg D, Ditah IC, Saeian K, Lalehzari M, Aronsohn A, Gorospe EC, et al. Changes in the prevalence of hepatitis $C$ virus infection, nonalcoholic steatohepatitis, and alcoholic liver disease among patients with cirrhosis or liver failure on the waitlist for liver transplantation. Gastroenterology. 2017; 152(5):1090-1099.e1 [cited 2019 mar 6]. Available from: http://www.ncbi. nlm.nih.gov/pubmed/28088461.

14. Benchimol El, Smeeth L, Guttmann A, Harron K, Moher D, Petersen I, et al. The REporting of studies conducted using observational routinely-collected health data (RECORD) statement. PLoS Med. 2015;12(10):e1001885[cited 2019 Mar 26]. Available from. https://doi.org/10.1371/journal.pmed.1001885.

15. Health I. IMS health : the health improvement network (THIN data) guide for researchers; 2017. [cited 2017 Mar 5]. Available from: http://www.epic-uk. org/our-data/our-data.shtml.

16. Blak BT, Thompson M, Dattani H, Bourke A. Generalisability of the health improvement network (THIN) database: demographics, chronic disease prevalence and mortality rates. Inform Prim Care. 2011;19(4):251-5.

17. Lewis JD, Schinnar R, Bilker WB, Wang X, Strom BL. Validation studies of the health improvement network (THIN) database for pharmacoepidemiology research. Pharmacoepidemiol Drug Saf. 2007;16, 393(4):-401 [cited 2019 Mar 6]. Available from: http://www.ncbi.nlm.nih.gov/pubmed/17066486.

18. Loomis AK, Kabadi S, Preiss D, Hyde C, Bonato V, St. Louis M, et al. Body mass index and risk of nonalcoholic fatty liver disease: two electronic health record prospective studies. J Clin Endocrinol Metab. 2016;101(3):945-52 [cited 2019 mar 6]. Available from: http://www.ncbi.nlm.nih.gov/ pubmed/26672639.

19. Kumarendran B, O'Reilly MW, Manolopoulos KN, Toulis KA, Gokhale KM, Sitch AJ, et al. Polycystic ovary syndrome, androgen excess, and the risk of nonalcoholic fatty liver disease in women: a longitudinal study based on a United Kingdom primary care database. Myers JE, editor. PLoS Med. 2018; 15(3):e1002542 [cited 2019 mar 6]. Available from: http://www.ncbi.nlm.nih. gov/pubmed/29590099.

20. Lewis JD, Bilker WB, Weinstein RB, Strom BL. The relationship between time since registration and measured incidence rates in the general practice research database. Pharmacoepidemiol Drug Saf. 2005;14(7):443-51 [cited 2019 mar 6]. Available from: http://www.ncbi.nlm.nih.gov/pubmed/15 898131

21. Sims EAH. Are there persons who are obese, but metabolically healthy? Metabolism. 2001;50(12):1499-504 [cited 2019 mar 6]. Available from: http:// www.ncbi.n/m.nih.gov/pubmed/11735101.

22. Karelis AD. Metabolically healthy but obese individuals. Lancet. 2008; 372(9646):1281-3 [cited 2019 mar 6]. Available from: http://www.ncbi.n/m. nih.gov/pubmed/18929889.

23. Blüher M. The distinction of metabolically 'healthy' from 'unhealthy' obese individuals. Curr Opin Lipidol. 2010;21(1):38-43 [cited 2019 mar 6]. Available from: http://www.ncbi.n/m.nih.gov/pubmed/19915462.

24. NHS Digital. Information and technology for better health and care. London: National Health Service; 2017. [Internet]. [cited 2019 Mar 7]. Available from: https://digital.nhs.uk/.

25. Expert Panel on Detection, Evaluation, and Treatment of High Blood Cholesterol in Adults. Executive summary of the third report of the National
Cholesterol Education Program (NCEP) expert panel on detection, evaluation, and treatment of high blood cholesterol in adults (adult treatment panel III). JAMA. 2001;285(19):2486-97 [cited 2019 mar 6] Available from: http://www.ncbi.nlm.nih.gov/pubmed/11368702.

26. World Health Organisation (WHO). Noncommunicable diseases global monitoring framework: indicator defintions and specifications. Geneva; 2014. [cited 2019 Mar 6]. Available from: https://www.who.int/nmh/ncd-tools/ indicators/GMF_Indicator_Definitions_Version_NOV2014.pdf.

27. Vision. In Practice Systems (INPS). About us -the health improvement network (THIN); 2016. [cited 2019 Mar 7]. Available from: https://www. visionhealth.co.uk/portfolio-items/the-health-improvement-network-thin/.

28. Xu C, Xu L, Miao M, Li Y, Yu C. Su1042 risk of developing nonalcoholic fatty liver disease among metabolically abnormal Normal-weight and metabolically healthy obese Chinese: a 7-year prospective study. Gastroenterology. 2015;148(4):S-1047 [cited 2019 Mar 6]Available from: https://linkinghub.elsevier.com/retrieve/pii/S0016508515335721.

29. Srikanthan K, Feyh A, Visweshwar H, Shapiro Jl, Sodhi K. Systematic review of metabolic syndrome biomarkers: a panel for early detection, management, and risk stratification in the west Virginian population. Int $J$ Med Sci. 2016 ;13(1):25-38. [cited 2019 mar 6]. Available from: http://www. ncbi.nlm.nih.gov/pubmed/26816492.

30. Jin S, Jiang S, Hu A. Association between obstructive sleep apnea and nonalcoholic fatty liver disease: a systematic review and meta-analysis. Sleep Breath. 2018;22(3):841-51 [cited 2019 mar 26]. Available from: http://www. ncbi.nlm.nih.gov/pubmed/29335916.

31. Mesarwi OA, Loomba R, Malhotra A. Obstructive sleep apnea, hypoxia, and nonalcoholic fatty liver disease. Am J Respir Crit Care Med. 2018;rccm. 201806-1109TR [cited 2019 mar 26]. Available from: http://www.ncbi.nlm. nih.gov/pubmed/30422676.

32. Peverill W, Powell L, Skoien R. Evolving concepts in the pathogenesis of NASH: beyond steatosis and inflammation. Int J Mol Sci. 2014;15(5):8591638 [cited 2019 mar 6]. Available from: http://www.ncbi.nlm.nih.gov/ pubmed/24830559.

33. Huh JH, Kim KJ, Kim SU, Han SH, Han K-H, Cha B-S, et al. Obesity is more closely related with hepatic steatosis and fibrosis measured by transient elastography than metabolic health status. Metabolism. 2017:66:23-31 [cited 2019 mar 6]. Available from: http://www.ncbi.nlm.nih.gov/pubmed/2 7923446.

34. Gómez-Ambrosi J, Catalán V, Rodríguez A, Andrada P, Ramírez B, Ibáñez P, et al. Increased Cardiometabolic risk factors and inflammation in adipose tissue in obese subjects classified as metabolically healthy. Diabetes Care. 2014;37(10):2813-21 [cited 2019 mar 6]. Available from: http://www.ncbi. nlm.nih.gov/pubmed/25011950.

35. Jin Y-J, Kim KM, Hwang S, Lee SG, Ha T-Y, Song G-W, et al. Exercise and diet modification in non-obese non-alcoholic fatty liver disease: analysis of biopsies of living liver donors. J Gastroenterol Hepatol. 2012;27(8):1341-7 [cited 2019 mar 6]. Available from: http://www.ncbi.n/m.nih.gov/ pubmed/22554085.

36. Appleton SL, Seaborn CJ, Visvanathan R, Hill CL, Gill TK, Taylor AW, et al. Diabetes and cardiovascular disease outcomes in the metabolically healthy obese phenotype: a cohort study. Diabetes Care. 2013;36(8):2388-94 [cited 2019 mar 6]. Available from: http://www.ncbi.n/m.nih.gov/pubmed/234 91523.

37. Soriguer F, Gutiérrez-Repiso C, Rubio-Martín E, García-Fuentes E, Almaraz MC, Colomo N, et al. Metabolically healthy but obese, a matter of time? Findings from the prospective Pizarra study. J Clin Endocrinol Metab. 2013; 98(6):2318-25 [cited 2019 mar 6]. Available from: http://www.ncbi.nlm.nih. gov/pubmed/23559087.

38. Bell JA, Hamer M, Sabia S, Singh-Manoux A, Batty GD, Kivimaki M. The natural course of healthy obesity over 20 years. J Am Coll Cardiol. 2015. 65(1):101-2 [cited 2019 mar 6]. Available from: http://www.ncbi.nlm.nih.gov/ pubmed/25572516.

39. Guo F, Garvey WT. Cardiometabolic disease risk in metabolically healthy and unhealthy obesity: stability of metabolic health status in adults. Obesity. 2016;24(2):516-25 [cited 2019 mar 6]. Available from: http://www.ncbi.nlm. nih.gov/pubmed/26719125.

40. Umano GR, Shabanova V, Pierpont B, Mata M, Nouws J, Tricò D, et al. A low visceral fat proportion, independent of total body fat mass, protects obese adolescent girls against fatty liver and glucose dysregulation: a longitudinal study. Int J Obes (Lond). 2019:43(4):673-82 [cited 2020 Jun 17]. Available from: http://www.nature.com/articles/s41366-018-0227-6. 
41. Ko YH, Wong TC, Hsu YY, Kuo KL, Yang SH. The correlation between body fat, visceral fat, and nonalcoholic fatty liver disease. Metab Syndr Relat Disord. 2017;15(6):304-11 [cited 2020 Jun 17]. Available from: http://www. liebertpub.com/doi/10.1089/met.2017.0001.

42. Younossi ZM, Stepanova M, Negro F, Hallaji S, Younossi Y, Lam B, et al. Nonalcoholic fatty liver disease in lean individuals in the United States. Medicine (Baltimore). 2012;91(6):319-27 [cited 2019 mar 6]. Available from: http://www.ncbi.nlm.nih.gov/pubmed/23117851.

43. Pan J-J, Fallon MB. Gender and racial differences in nonalcoholic fatty liver disease. World J Hepatol. 2014;6(5):274 [cited 2019 mar 6]. Available from: http://www.ncbi.nlm.nih.gov/pubmed/24868321.

\section{Publisher's Note}

Springer Nature remains neutral with regard to jurisdictional claims in published maps and institutional affiliations.

Ready to submit your research? Choose BMC and benefit from:

- fast, convenient online submission

- thorough peer review by experienced researchers in your field

- rapid publication on acceptance

- support for research data, including large and complex data types

- gold Open Access which fosters wider collaboration and increased citations

- maximum visibility for your research: over $100 \mathrm{M}$ website views per year

At BMC, research is always in progress.

Learn more biomedcentral.com/submissions 